\title{
Differences in Movement Speed Before and After a Split-Step Between Professional and Junior Tennis Players
}

\author{
by

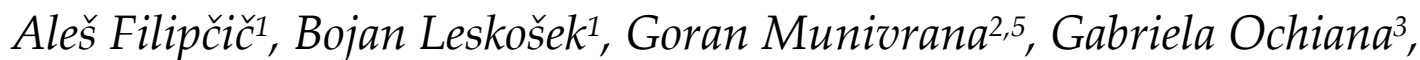 \\ Tjaša Filip̌čič ${ }^{4}$
}

\begin{abstract}
This study investigated tennis players' speed before, during and after the split-step, deceleration before and acceleration after the split-step in four different stroke groups in three age categories. Seven male professional, eleven male and ten female junior tennis players were recorded with video cameras at official tournaments. Using the SAGIT system, we gathered data on 8,545 split-steps. Tennis players performed a split-step in $82.9 \%$ of cases. A tennis player's speed, deceleration and acceleration were measured $0.2 \mathrm{~s}$ before and after the split-step. Differences between categories and stroke groups for each of the five variables were analyzed with a two-way ANOVA. The differences between the groups of players were generally much higher in the speed before, during and after the split-step than in the deceleration before and acceleration after the split-step. Most of these differences were observed between the various stroke groups. These results suggest that players use three types of movement while performing a split-step. In the first type, which is typical of serving and returning, the speed before, during and after the split-step is lower (0.55 to $1.2 \mathrm{~m} / \mathrm{s})$. The second type of movement is characteristic of baseline strokes where tennis players achieve higher speed than in the first type $(0.7$ to $1.66 \mathrm{~m} / \mathrm{s})$. The third type occurs in strokes where a tennis player is moving or already at the net $(0.78$ to 1.9 $\mathrm{m} / \mathrm{s}$ ). Movement in tennis is an area that requires constant development in terms of designing and upgrading movement patterns, increasing speed and practice in specific game situations.
\end{abstract}

Key words: tennis, movement analysis, game situations.

\section{Introduction}

Tennis is a dynamic, open-skill sport that requires decision-making, movement and hitting skills under constant time pressure (Nieminen et al., 2014; O'Donoghue and Ingram, 2001). Highlevel tennis players make an average of four directional changes per point; otherwise, the number of changes ranges from 1 to 15 in long rallies (Kovacs, 2009). A directional change is on average performed every $4 \mathrm{~m}$ (Pieper et al., 2007). Tennis players execute the majority of strokes
$(80 \%)$ within the so-called comfort zone, i.e. with movements over a distance of up to $3 \mathrm{~m}$ (Over and O'Donoghue, 2008). Most (71.8\%) movements are performed laterally, less than $20 \%$ of movements are in a forward direction and less than $8 \%$ of them are in a backward direction. Almost half of the strokes (44.7\%) are performed under time pressure (Weber et al., 2007). On a grass surface, two-thirds $(66.6 \%)$ of movements before the stroke start with a split-step (Hughes

\footnotetext{
1 - University of Ljubljana, Faculty of Sport, Ljubljana, Slovenia.

2 - University of Split, Faculty of Kinesiology, Split, Croatia.

3 - "Vasile Alecsandri" University of Bacau, Faculty of Movement, Sports, and Health Sciences, Bacau, Romania.

4 - University of Ljubljana, Faculty of Education, Ljubljana, Slovenia.

5 - Sports Science and Medical Committee, International Table Tennis Federation, Lausanne, Switzerland
} 
and Meyers, 2005).

Efficient movement depends on agility and speed. Speed or velocity describes the rate at which an athlete moves from one location to another. In addition to movement speed, there are important features like acceleration speed, reaction speed, frequency speed, and single movement acceleration. Previous studies have established that speed is very important in determining competitive successfulness in junior tennis (Filipčič and Filipčič, 2005).

The split-step is a preparatory movement most often performed by a player just before the opponent's stroke (Aviles et al., 2002). It represents an integral part of preparing for a volley, return of serve or groundstroke. It places the quadriceps muscle in a stretch, permitting the storage and subsequent release of energy to enhance quick movement in preparation for the following stroke (Elliott, 2006). The split-step enables a faster reaction (Tatsuo et al., 2005), a fast start of the movement (Uzu et al., 2009) and an effective change in direction (Bloomfield at al., 1994; Salonikidis and Zafeiridis, 2008). Similar to the split-step for starting the movement is the importance of the first step for continuing the movement by initiating a forward movement, increasing force and power at push-off and improving sprint performance over short distances (Frost and Cronin, 2011). Jacobs and Van Ingen Schenau (1992) indicated that the aim of the motor system in the first step of sprinting is to control the transformation of joint angular accelerations into an increase in velocity at the beginning in a horizontal direction.

The ability to quickly and explosively execute the first step following the split-step is crucial for an effective movement. Lamond et al. (1996) found that players on the return of the serve used a lateral step (i.e. jab step) with intent to increase their base of support and become more stable prior to the movement. Moreover, the same starting movement is often used by tennis players on the baseline when executing strokes in the comfort zone. Bragg and Andriacchi (2001) found that tennis players on the baseline, after opening their hips and shoulders, used a lateral reaction step. This step enables tennis players to accelerate faster after the split-step. This kind of starting movement was found to be more effective when tennis players moved forwards or backwards and always made the first step in the opposite direction of the movement (Frost and Cronin, 2011). According to Parsons and Jones (1998), a quick lateral movement is crucial when a tennis player must react immediately and change the direction. Komi (2000) indicated that this kind of movement was more effective than a pure concentric muscle action. Roetert et al. (2003) defined the split-step as a tennis-specific movement pattern where a stretch-shortening cycle muscle action occurred.

Tennis players use a lateral reaction step for sprints over distances from 2.5 to 6 meters. For movements in situations of short or wide balls, a fast and explosive movement in the first two or three steps after the split-step is also typical (Crespo and Miley, 1998). Otherwise, an efficient movement consists of various types of steps, such as the multi-directional shuffle, side-step and small adjustment steps, which enables a tennis player to carry out strokes with good balance in both offensive and defensive situations (Bourquin, 2003).

In previous studies, the speed had been measured by general movement tests, conducted outside a tennis court. Therefore, our aim was to measure the movement speed during a tennis match, and more specifically, to measure the tennis player's speed before, during and after the split-step. We were most interested in determining whether there were any differences in deceleration before and acceleration after the split-step in various types of strokes as well as whether there were any differences in tennis players' movement regarding their sex, age and sports level.

The purposes of the present study were: 1) to examine speed before, during and after the split-step, deceleration before and acceleration after the split-step in four types of strokes (after the serve, return of the serve, baseline strokes and other strokes); and 2) to find differences in three categories of tennis players (ATP players, boys and girls 14 \& under).

\section{Material and Methods}

\section{Sample of matches and participants}

The Faculty of sport Human Ethics Committee at the University of Ljubljana approved the experimental design and protocol prior to data collection. The sample included 15 
matches played at a national tournament, in which 14 \& under boys (B14) and girls (G14) participated. For the ATP players, also the data of 15 matches played at an international tournament were collected. All tennis players $(n=28)$ were considered as highly trained and ranked on a national (14 \& under players) or professional ranking list (ATP players). All the matches took place on a Rebund Ace GS surface on an indoor tennis court (Table 1).

\section{Procedure}

During the tournaments, all matches were recorded with fixed SVHS video cameras (Ultrak CCD Color KC 7501 CP) with a frequency of capturing input images of $25 \mathrm{~Hz}$. Each camera was fastened to the ceiling and therefore its wideangled lens (Ultrak KL 28141s $2.8 \mathrm{~mm}$, Japan) covered an entire half of the court. The video recordings were digitized using the Video DC30* video digitizer hardware (Miro, Germany) with resolution of $384 \times 576$ at a $2 \mathrm{MB}$ s- 1 data rate, while processing was carried out at resolution of $384 \times 288$ pixels.

The digital images were processed with the SAGIT/TENNIS tracking system using a computer vision method (Perš et al., 2002; Vučković et al., 2010). The conversion into numerical data entailed the following steps: (1) recording tennis matches; (2) calibration of the recordings (time and space calibration); (3) data processing with the SAGIT/TENNIS tracking system (notation of players' movement, splitsteps, strokes, outcomes and game phases) and exporting into a database; (4) organizing the data with a combination of SQL statements and exporting it to Microsoft Excel; and (5) processing the data with the statistical program SPSS 23 for Windows.

\section{Data treatment}

Using the SAGIT system, we gathered data on 18,856 actions (10,311 strokes; 8,545 splitsteps). For further analyses, we selected actions $(82.9 \%)$ in which a tennis player performed a split-step and it was possible to precisely determine the timing of that split-step. Split-steps were organized according to a player's category (ATP players, boys and girls 14 \& under) and the type of the previous stroke: serve (stroke group 1), return of the serve (stroke group 2), forehand and backhand on the baseline (stroke group 3) and other strokes (stroke group 4). Among other strokes, we placed the drop shot, volley, drive volley, slice overhead smash on the forehand and backhand side. A tennis player's speed was measured during the split-step in five frames $(0.2$ s) before and after the split-step. Based on previous measuring in $0.2 \mathrm{~s}$ or in five frames after the split-step, an individual tennis player also made the lateral step. The speed before and after the split-step were the average speeds in five frames. Tennis player's deceleration before and acceleration after the split-step were also measured within $0.2 \mathrm{~s}$. The change in speed before and after the split-step was calculated using the following formula: deceleration before the splitstep $=$ speed during the split-step / speed before the split-step, and acceleration after the split-step = speed during the split-step / speed after the split-step.

Statistics

Basic distributional statistics were computed for all five split-step variables (speed before, during and after the split-step, deceleration before the split-step and acceleration after the split-step) for each stroke and stroke group joined and separated for each category (B14, G14, ATP). Differences between categories and stroke groups for each of the five variables were analyzed by a two-way ANOVA. The effect size of each ANOVA's factor and their interaction was evaluated by eta squared. Differences between category pairs were post-hoc evaluated by the Tukey (HSD) and Scheffé tests; $p$-values below 0.05 were considered statistically significant. The analysis was performed with IBM SPSS Statistics Version 23.

\section{Results}

Distributional statistics of speed during the split-step are shown in Table 2. The smallest mean values were observed in stroke group 1, followed by stroke groups 2 and 3 . In stroke group 4, the various strokes had quite different means, but usually much higher than strokes from the other stroke groups. The variability in stroke groups 3 and 4 was much higher than in stroke groups 1 and 2 . The distribution of speed during the split-step for strokes in the first three stroke groups was moderately positive (right asymmetrical), while in stroke group 4 most strokes exhibited an almost symmetrical distribution. 
Deceleration before and acceleration after the split-step had a similar distribution in all four stroke groups and all three categories, while the means in the other three variables (speed before, during and after the split-step) were usually smallest in stroke group 1, followed by stroke groups 2, 3 and 4 (Table 3). In those three variables, the variability was also higher in groups 3 and 4 than in groups 1 and 2 .

Table 1

Subjects' characteristics.

\begin{tabular}{cccccc}
\hline Group & N & Age (years) & Height $(\mathrm{cm})$ & Mass $(\mathrm{kg})$ & Ranking \\
\hline Boys 14\&U & 11 & $13.8 \pm 0.7$ & $176 \pm 7.5$ & $67.6 \pm 11$ & $7(1-17)^{*}$ \\
Girls 14\&U & 10 & $14.1 \pm 0.5$ & $162.2 \pm 8.4$ & $52.1 \pm 4.4$ & $4.6(1-18)^{*}$ \\
ATP players & 7 & $18.3 \pm 4.7$ & $181.4 \pm 9.5$ & $75 \pm 7.7$ & $380(121-1020)^{* *}$ \\
\hline
\end{tabular}

*national ranking ${ }^{* *}$ ATP ranking

Table 2

Distributional statistics of speed during the split step by the stroke and stroke type

\begin{tabular}{|c|c|c|c|c|c|}
\hline Stroke type & Stroke & $\mathbf{n}$ & Mean & SD & Skewness \\
\hline \multirow{3}{*}{1 (serve) } & $1^{\text {st }}$ serve & 1557 & .78 & .48 & .89 \\
\hline & $2^{\text {nd }}$ serve & 605 & .85 & .45 & .78 \\
\hline & Group 1 total & 2162 & .80 & .47 & .85 \\
\hline \multirow{5}{*}{2 (return of serve) } & $1^{\text {st }}$ serve backhand return & 392 & 1.02 & .55 & 1.51 \\
\hline & $1^{\text {st }}$ serve forehand return & 494 & 1.05 & .46 & 1.10 \\
\hline & $2^{\text {nd }}$ serve backhand return & 290 & 1.03 & .44 & .66 \\
\hline & $2^{\text {nd }}$ serve forehand return & 216 & 1.12 & .48 & 1.13 \\
\hline & Group 2 total & 1392 & 1.05 & .49 & 1.20 \\
\hline \multirow{3}{*}{3 (baseline strokes) } & Backhand topspin & 1970 & 1.25 & .82 & 1.04 \\
\hline & Forehand topspin & 2426 & 1.31 & .83 & .85 \\
\hline & Group 3 total & 4396 & 1.28 & .82 & .93 \\
\hline \multirow{11}{*}{4 (other strokes) } & Backhand drop shot & 15 & 1.75 & .94 & .38 \\
\hline & Backhand drive volley & 6 & 2.29 & 1.03 & -.28 \\
\hline & Backhand slice & 355 & 1.62 & .87 & .58 \\
\hline & Backhand Smash & 1 & 2.04 & & \\
\hline & Backhand volley & 43 & 2.37 & .87 & -.56 \\
\hline & Forehand drop shot & 11 & 1.95 & .78 & 2.24 \\
\hline & Forehand drive volley & 19 & 2.29 & .83 & -.04 \\
\hline & Forehand slice & 81 & 1.64 & .85 & .35 \\
\hline & Forehand smash & 18 & 1.72 & .84 & -.09 \\
\hline & Forehand volley & 46 & 2.30 & 1.05 & .11 \\
\hline & Group 4 total & 595 & 1.77 & .92 & .416 \\
\hline
\end{tabular}


Table 3

Mean \pm SD for split-step variables by category and stroke group

\begin{tabular}{cccccc}
\hline & & \multicolumn{3}{c}{ Stroke group } \\
Parameter & Category & 1 & 2 & 3 & 4 \\
\hline Speed during the split-step & ATP & $1.13 \pm 0.47$ & $1.07 \pm 0.60$ & $1.55 \pm 0.97$ & $1.86 \pm 0.97$ \\
& B14 & $0.64 \pm 0.38$ & $1.01 \pm 0.43$ & $1.18 \pm 0.74$ & $1.54 \pm 0.78$ \\
& G14 & $0.58 \pm 0.34$ & $1.07 \pm 0.42$ & $1.27 \pm 0.81$ & $1.89 \pm 0.91$ \\
\hline Speed before the split-step & ATP & $0.97 \pm 0.44$ & $1.04 \pm 0.57$ & $1.57 \pm 0.90$ & $1.82 \pm 0.92$ \\
& B14 & $0.58 \pm 0.35$ & $1.08 \pm 0.40$ & $1.15 \pm 0.70$ & $1.49 \pm 0.78$ \\
& G14 & $0.55 \pm 0.31$ & $1.14 \pm 0.37$ & $1.26 \pm 0.77$ & $1.79 \pm 0.86$ \\
\hline Speed after the split-step & ATP & $1.23 \pm 0.51$ & $1.24 \pm 0.63$ & $1.66 \pm 1.00$ & $1.90 \pm 1.02$ \\
& B14 & $0.78 \pm 0.45$ & $1.17 \pm 0.50$ & $1.29 \pm 0.77$ & $1.62 \pm 0.79$ \\
step & G14 & $0.70 \pm 0.39$ & $1.20 \pm 0.53$ & $1.36 \pm 0.82$ & $1.93 \pm 0.95$ \\
\hline Deceleration before the split- & ATP & $1.23 \pm 0.37$ & $1.07 \pm 0.34$ & $1.01 \pm 0.37$ & $1.04 \pm 0.29$ \\
& B14 & $1.22 \pm 0.52$ & $0.95 \pm 0.29$ & $1.08 \pm 0.42$ & $1.08 \pm 0.31$ \\
step & G14 & $1.16 \pm 0.55$ & $0.93 \pm 0.26$ & $1.04 \pm 0.36$ & $1.08 \pm 0.26$ \\
\hline & ATP & $0.95 \pm 0.31$ & $0.88 \pm 0.26$ & $0.99 \pm 0.44$ & $1.01 \pm 0.33$ \\
& B14 & $0.88 \pm 0.36$ & $0.91 \pm 0.31$ & $0.97 \pm 0.39$ & $1.00 \pm 0.38$ \\
& G14 & $0.88 \pm 0.36$ & $0.95 \pm 0.30$ & $0.97 \pm 0.38$ & $1.04 \pm 0.42$ \\
\hline
\end{tabular}

\section{Table 4}

Two-way ANOVA for the differences between category and stroke groups in five split-step variables

\begin{tabular}{|c|c|c|c|c|}
\hline Source & Parameter & $\mathrm{F}$ & $p$ & $\begin{array}{c}\text { Eta } \\
\text { squared }\end{array}$ \\
\hline \multirow{5}{*}{ Category } & Speed during the split-step & 94.66 & $<0.01$ & $1.8 \%$ \\
\hline & Speed before the split-step & 83.49 & $<0.01$ & $1.6 \%$ \\
\hline & Speed after the split-step & 80.01 & $<0.01$ & $1.6 \%$ \\
\hline & Deceleration before the split-step & 2.65 & 0.07 & $0.1 \%$ \\
\hline & Acceleration after the split-step & 2.07 & 0.13 & $0.0 \%$ \\
\hline \multirow{5}{*}{ Stroke Group } & Speed during the split-step & 444.04 & $<0.01$ & $13.0 \%$ \\
\hline & Speed before the split-step & 568.50 & $<0.01$ & $16.1 \%$ \\
\hline & Speed after the split-step & 359.68 & $<0.01$ & $10.9 \%$ \\
\hline & Deceleration before the split-step & 104.71 & $<0.01$ & $3.5 \%$ \\
\hline & Acceleration after the split-step & 28.90 & $<0.01$ & $1.0 \%$ \\
\hline \multirow{5}{*}{$\begin{array}{c}\text { Category }{ }^{*} \text { Stroke } \\
\text { Group }\end{array}$} & Speed during the split-step & 20.26 & $<0.01$ & $1.2 \%$ \\
\hline & Speed before the split-step & 22.63 & $<0.01$ & $1.3 \%$ \\
\hline & Speed after the split-step & 16.06 & $<0.01$ & $1.0 \%$ \\
\hline & Deceleration before the split-step & 7.70 & $<0.01$ & $0.5 \%$ \\
\hline & Acceleration after the split-step & 4.13 & $<0.01$ & $0.3 \%$ \\
\hline \multirow{5}{*}{ Corrected Model } & Speed during the split-step & 156.74 & $<0.01$ & $16.8 \%$ \\
\hline & Speed before the split-step & 187.58 & $<0.01$ & $19.5 \%$ \\
\hline & Speed after the split-step & 127.80 & $<0.01$ & $14.1 \%$ \\
\hline & Deceleration before the split-step & 34.13 & $<0.01$ & $4.2 \%$ \\
\hline & Acceleration after the split-step & 10.56 & $<0.01$ & $1.3 \%$ \\
\hline
\end{tabular}


Differences between professional male and female tennis players in their speed before, after and during the split-step were greater and significant, while the speed change coefficient before and after the split-step was non-significant. Most of these differences are attributable to the effect of the stroke group. Although the effects of category and category* stroke group interaction were in most cases (marginally) significant, they were much lower (Table 4). As expected, due to differences in their physical characteristics, movement abilities and patterns, the ATP tennis players achieved a significantly faster movement speed before, after and during the split-step after serves, returns and baseline shots in comparison to junior tennis players. All three groups of players achieved higher speed change coefficients before the split-step that was at the time of deceleration, as opposed to the time of acceleration after the split-step. Players in all three groups accelerated most in other strokes and decelerate most in both serves.

All pairwise differences between groups were significant for both (stroke group, category) factors in the speed before and during the splitstep variables. The same was true for all speed differences after the split-step, except for the difference between categories B14 and G14.

In deceleration before the split-step, significant differences in the speed change coefficient were noted in both the category of tennis players as well as the category of stroke groups. The exception were the differences between the ATP players and boys $14 \&$ under in stroke groups 3 and 4 . In acceleration and deceleration, differences in the speed change coefficient were only observable between stroke groups 1 and 2, and between stroke groups 3 and 4. Post-hoc analysis between category pairs evaluated by the Tukey (HSD) and Scheffé tests showed that only between B14 and G14 there were non-significant differences in speed after the split-step.

\section{Discussion}

The aim of this study was to analyze players running speed and acceleration before, after and during a split-step in four different game situations as well as in three age groups of a different performance level. Since tennis is a dynamic, fast and unpredictable game, tennis players' movement efficiency is influenced by spatial and temporal factors. These are first determined by five ball characteristics i.e. direction, depth, height, rotation and speed. In addition, the movement and execution of the stroke is influenced by a player's own position upon an opponent's stroke, distance from the intended position of the following stroke, desired direction of the executed stroke and, from a tactical point of view, the opponent's movement and positioning during execution of the stroke. A tennis player's ability to coordinate all of these movements at high speed to permit effective stroke execution therefore becomes a significant performance-determining factor (Elliott, 2006). Tennis strokes are divided into five game situations according to tactical intent: serving, returning (receiving), playing on the baseline, approaching and net game, and defending (playing against a player at the net). The movement cycle in tennis is defined by five phases: positioning, movement to the ball, placement around the ball, hitting stance and recovery (Crespo and Miley, 1998).

In our study, the strokes were divided into four groups. The first three corresponded to applicable game situations, while in the fourth, due to a smaller number of repetitions (frequency), we joined both offensive and defensive strokes. The game situation in which a player is involved, has the greatest influence on both differences in speed before, after and during the split-step as well as on the speed change coefficient values in deceleration and acceleration.

The movement efficiency of tennis players is affected by many factors, including the speed, placement and effectiveness of opponent's strokes (James and Bradley, 2004). On the basis of analysis of match performance indicators, FernandezGarcia et al. (2015) detected that on a hard court, professional tennis players achieved a higher share of points than junior players in both firstserve percentage as well as in first and second serve points won and achieved on average one more ace per set. Speed and accuracy of the serve also affect the movement style and execution of the return, which indirectly influences the efficiency of the returning player. In our study, junior tennis players achieved lower movement speeds while returning, which had an indirect influence on more return points won, break points 
per set, break points won and breaks per set. Professional tennis players were more effective in play after the serve and return as on average they made 10 winners and unforced errors per set, while junior players made slightly less than 2 . Again, the decisive reason was the higher speed of the strokes that mainly affected differences in tennis players' speed before the split-step. ATP tennis players entered the split-step with a higher speed and could perform movement in eccentricconcentric conditions of muscle functioning.

The lowest average speeds tennis players achieved were after the first and second serve $(0.8$ $\mathrm{m} / \mathrm{s})$, slightly higher after the return of the first and second serve $(1.05 \mathrm{~m} / \mathrm{s})$, even higher when playing forehand and backhand on the baseline $(1.28 \mathrm{~m} / \mathrm{s})$ and the highest in other strokes $(1.77$ $\mathrm{m} / \mathrm{s})$. The differences in speed during the splitstep after the first $(0.78 \mathrm{~m} / \mathrm{s})$ and second serve $(0.85 \mathrm{~m} / \mathrm{s})$ were small, but expected. Players risk more with the first serve, considering that the speed of the first serve of ATP players is on average $20 \%$ faster than the speed of the second serve (Vaverka, 2010), which also influences a player's movement when executing the return. Consequently, after their stroke servers have more time to prepare and do not need to develop a higher movement speed, which also influences the lower speed during the split-step. This is consistent with the finding (Hughes and Meyers, 2005) that a player's initial movement while serving is a jump, followed by a neutral split-step, which allows the tennis player an optimal balanced position and continuation of their movement.

In response to a return, the tennis players also achieved a lower speed during the split-step after the return of the first serve. In comparison to the return of the second serve, also due to the lower serve speed returners had more chances to put their opponent under time pressure and force a faster movement. The movement speed during the split-step in a baseline game was higher after a forehand $(1.31 \mathrm{~m} / \mathrm{s})$ than after a backhand $(1.25$ $\mathrm{m} / \mathrm{s})$, confirming the assumption that tennis players were more aggressive with their forehand. In other strokes, players achieved highest speed during the split-step in all strokes inside the court or at the net (volley, drive volley), again pointing to the fact that these kinds of strokes are executed very dynamically and often in a movement towards the net, indirectly influencing the execution of the split-step.

We can determine three types of movement in terms of tennis player's speed before, during and after the split-step. In the first type, typical for serving and returning, the speed before, during and after the split-step is lower and ranges from 0.55 to $1.2 \mathrm{~m} / \mathrm{s}$. The lower speed in comparison to other game situations is due to the shortest movement route to make the stroke and the limited time a tennis player has available to execute the split-step and stroke. A tennis player's speed is constantly growing and is the lowest before the split-step and highest after the splitstep.

The second type of movement is characteristic of baseline strokes where, however, tennis players achieve higher speed than in the first type, ranging from 0.7 to $1.66 \mathrm{~m} / \mathrm{s}$. Speed is high in all three phases of the split-step, with a visible trend of speed decreasing over time due to the tennis player's intention to calm down during the split-step as most as possible, allowing $\mathrm{him} /$ her to efficiently continue the movement. In a game situation where both players are on the baseline, tennis players have more time to execute a stroke than when serving and returning.

The third type occurs in strokes when a tennis player is moving or is already at the net. In this type of movement, tennis players achieve a speed ranging from 0.78 to $1.9 \mathrm{~m} / \mathrm{s}$. This type is essentially similar to the first one, although the starting speed (before the split-step) is higher because in executing a volley, drive volley or overhead smash, a tennis player wants to preserve as much momentum as possible and hit the ball in motion through the stroke. Efficient movement towards the net and at the net is also described by instructions of tennis coaches who advise tennis players to: lower their center of gravity to allow the player to move quickly and powerfully, step into the ball when hitting it and follow the ball's path after hitting an approach shot or volley (Crespo and Miley, 1998).

One limitation of our work may lie in the correlation between movement speed and splitstep timing, which also considerably influences a tennis player's movement. Appropriate split-step timing could also indirectly affect the movement speed of a tennis player before, during and after the split-step. One aspect not included in this 
study is the content of the movement, that is the pattern with which a tennis player moves before and after the split-step and the distance, he/she covers in between two shots. The distance a tennis player covers from the previous stroke to the split-step and after that to the next stroke influences both the type and speed of movement. Finally, another aspect not covered is how characteristics of execution of the split-step influence the efficiency of strokes and the success of tennis players.

The split-step, as an integral part of preparing for the next stroke, positively influences tennis player's efficiency of movement from both neuromuscular and perceptive aspects; it changes according to different game situations and is closely related to the opponent's ball characteristics. Our findings revealed differences between the sports level and age of players in particular types of movement.
Tennis players use various types of movement in different game situations that allows to draw at least three main conclusions. Firstly, each tennis practice has to include all game situations - from serving and returning to playing at the baseline as well as approaching and playing at the net. Secondly, movement, especially the split-step, requires development in open game situations with "live ball" rallies. Thirdly, the development of movement patterns outside a tennis court is required, where the emphasis should be on the explosiveness and speed of the tennis movement.

Movement in tennis is an area that requires constant development in terms of both designing and upgrading movement patterns as well as increasing speed, but especially in choosing game situations, which a tennis coach should include in everyday practice.

\section{References}

Aviles C, Benguigui N, Beaudoin E, Godart F. Developing early perception and getting ready for action on the return of serve, CSSR, 2002; $28: 6-8$

Bloomfield J, Ackland TR, Elliot BC. Applied anatomy and biomechanics in sport. Melbourne: VIC: Blackwell Scientific; 1994

Bourquin O. Coordination. In Reid M, Quinn A, Crespo M. (Ed.), ITF Strength and Conditioning for Tennis. London: International Tennis Federation Ltd., 71-77; 2003

Bragg RW, Andriacchi TP. The lateral reaction step in tennis footwork. 19th International Symposium on Biomechanics in Sports, San Francisco; 2001

Crespo M, Miley D. Advanced Coaches Manual. London: International Tennis Federation; 1998

Elliott B. Biomechanics and tennis. Br J Sports Med, 2006; 40: 392-396

Fernandez-Garcia AI, Torres-Luque G, Sánchez-Pay A, Cabello-Manrique D. Differences in game statistics between men and junior boys in different surfaces. In Kondrič M, Xiaopeng Z, Dandan X. (Ed.), 14th ITTF Sports Science Congress and 5th World Racquet Sports Congress Suzhou, Suzhou: International table tennis association, 105; 2015

Filipčič A, Filipčič T. The influence of tennis motor abilities and anthropometric measures on the competition successfulness of 11 and 12 year-old female tennis players. Acta Univ. Palacki, 2005; 35: 35-41

Frost DM, Cronin JB. Stepping back to improve sprint performance: a kinetic analysis of the first step forwards. J Strength Cond Res, 2011; 10: 2721-2728, Retrieved May 15, 2016 from: http://journals.lww.com/nscajscr/Abstract/2011/10000/Stepping_Back_to_Improve_Sprint_Performance_A.11.aspx

Hughes M, Meyers R. Movement patterns in elite men's singles tennis. Int J Perform Anal Sport, 2005; 5: 110134

Jacobs R, van Ingen Schenau GJ. Intermuscular coordination in a sprint push-off. J Biomech, 1992; 25: 953-965

James N, Bradley C. Disguising one's intentions: The availability of visual cues and situational probabilities when playing against an international level squash player. In Lees A, Kahn JF, Maynard IW. (Ed.), Science and Racket Sports III Abingdon, Oxon: Routledge, 247-252; 2004

Komi PV. Stretch-shortening cycle: A powerful model to study normal and fatigued muscle. J Biomech, 2000; 
33: $1197-1206$

Kovacs M. Movement for Tennis: The Importance of Lateral Training. Strength Cond J, 2009; 31: 77-85, Retrieved May 15, 2016 from: http://journals.lww.com/nscascj/Abstract/2009/08000/Movement_for_Tennis_The_Importance_of_Lateral.9.aspx

Lamond F, Lowdon B, Davis K. Determination of the quickest footwork for teaching return of the tennis serve. ACHPER Healthy Lifestyles Journal, 1996; 43: 5-8

Nieminen MJ, Piirainen JM, Salmi JA, Linnamo V. Effects of neuromuscular function and split step on reaction speed in simulated tennis response. Eur J Sport Sci, 2014; 14: 318-326

O'Donoghue PG, Ingram B. A notational analysis of elite tennis strategy. J Sports Sci, 2001; 19: 107-115

Over S, O'Donoghue PG. What's the point? Tennis analysis and why. CSSR 2008; 15: 19-21, Retrieved May 15, 2016 from: http://en.coaching.itftennis.com/media/113918/113918.pdf

Parsons LS, Jones MT. Development of speed, agility, and quickness for tennis athletes. Strength Cond J, 1998; 20: $14-19$

Perš J, Bon M, Kovačič S, Šibila M, Dežman B. Observation and analysis of large-scale human motion. Hum Mov Sci, 2002; 21: 296-311

Pieper S, Exler T, Weber K. Running speed loads on clay and hard courts in world class tennis. STMS, 2007; 12: $14-17$

Roetert P, Ellenbecker T, Chu D. Movement mechanics. In Reid M, Quinn A, Crespo M, (Ed.), ITF Strength and Conditioning for Tennis, London: International Tennis Federation Ltd., 165-174; 2003

Salonikidis K, Zafeiridis A. The effects of plyometric, tennis-drills, and combined training on reaction, lateral and linear speed, power, and strength in novice tennis players. J Strength Cond Res, 2008; 1: 182-191,

Retrieved May 15, 2016 from:

http://journals.lww.com/nscajscr/Abstract/2008/01000/The_Effects_of_Plyometric,_Tennis_Drills,_and. 27.aspx

Tatsuo M, Takeo N, Masahiro O, Yoshimori K, Toshiyasu N, Hideo M, Yoshiaki T. Quick movement in tennis footwork: Effectiveness of the split step. Japanese Society for Orthopaedic Biomechanics, Hikarigaoka, Tokyo 363-367; 2005

Uzu R, Shinya M, Oda S. A split-step shortens the time to perform a choice reaction step-and-reach movement in a simulated tennis task. J Sports Sci, 2009; 27: 1233-1340

Vaverka F. Grand Slam 2008-2009. Olomouc: Univerzita Palackeho v Olomouci, Fakulta telesne kultury; 2010

Vučković G, Perš J, James N, Hughes M. Measurement error associated with the SAGIT/Squash computer tracking software. Eur J Sport Sci, 2010; 10: 129-140

Weber K, Pieper S, Exler T. Characteristics and significance of running speed at the Australian Open 2006 for training and injury prevention. STMS, 2007; 12: 14-17, Retrieved May 15, 2016 from: http://tennismedicine.org/

\section{Corresponding author:}

Associate professor Aleš Filipčič, Ph. D.

University of Ljubljana, Faculty of sport, Gortanova 22, SI1000 Ljubljana, Slovenia, EU

Phone: +386 41704 876; Fax. +386 15207760

E-mail: ales.filipcic@fsp.uni-lj.si 